\title{
Upstream rheotaxis of catalytic Janus spheres
}

\author{
Priyanka Sharan ${ }^{1}$ and Zuyao Xiao ${ }^{1}$ and Viviana Mancuso ${ }^{2}$ and \\ William E. Uspal ${ }^{2 *}$ and Juliane Simmchen ${ }^{1 *}$ \\ ${ }^{1}$ Chair of Physical Chemistry, TU Dresden, 01062 Dresden, Germany, \\ ${ }^{2}$ Department of Mechanical Engineering, University of Hawai'i at Mānoa, Honolulu, HI, 96822, USA,
}

*To whom correspondence should be addressed; E-mail: juliane.simmchen@tu-dresden.de

Fluid flow is ubiquitous in many environments that form habitats for microorganisms. The tendency of organisms to navigate towards or away from flow is termed rheotaxis.Therefore, it is not surprising that both biological and artificial microswimmers show responses to flows that are determined by the interplay of chemical and physical factors. In particular, to deepen understanding of how different systems respond to flows, it is crucial to comprehend the influence played by swimming pattern. In recent studies, pusher-type Janus particles exhibited cross-stream migration in externally applied flows. Earlier, theoretical studies predicted a positive rheotactic response for puller-type spherical Janus micromotors. To compare to a different swimmer, we introduce $\mathrm{Cu} @ \mathrm{SiO}_{2}$ micromotors that swim towards their catalytic cap. Based on experimental observations, and supported by flow field calculations using a model for self-electrophoresis, we hypothesize that they behave effectively as a puller-type system. We investigate the effect of externally imposed flow on these spherically symmetrical $\mathrm{Cu} @ \mathrm{SiO}_{2}$ active Janus colloids, and we indeed 
observe a steady upstream directional response. Through a simple squirmer model for a puller, we recover the major experimental observations. Additionally, the model predicts a unique "jumping" behaviour for puller-type micromotors at high flow speeds. Performing additional experiments at high flow speeds, we capture this phenomenon, in which the particles "roll" with their swimming axes aligned to the shear plane, in addition to being dragged downstream by the fluid flow.

\section{Introduction}

A key feature in microorganisms is an ability termed "taxis," i.e., the skill to navigate away from or towards certain stimuli. This ability of microorganisms to recognise changes in the environment and optimize their movement direction towards favorable areas drastically influences their survival rate ${ }^{[1,2]}$. Tactic behaviour in living systems can be triggered by various physicochemical changes, for instance chemical (chemotaxis) ${ }^{[3]}$, light (phototaxis) ${ }^{[4,5]}$, and temperature (thermotaxis ${ }^{[6]}$. Microorganisms in aquatic and terrestrial habitats undoubtedly experience another interesting environmental cue, which is the presence of water currents. The motile cells can either migrate up or down the flow, and this response is termed rheotaxis. While in fish and other macroscale aquatic organisms, the rheotaxis reaction is based on their sense organs and internal processing of sensory information, in case of microorganisms such as spermatozoa, it is believed to be purely physical in origin ${ }^{[7,8]}$. Fluid flow from the oviduct to the uterus is considered to provide the long range navigation mechanism behind the upstream motion of sperm cells, which assists them in traveling thousands of their body lengths in the complex genital tract of females. Similarly, E. Coli exploit hydrodynamic interactions to cause upstream bacterial infections in the human urinary tract ${ }^{[9]}$.

In the past few years, various artificial microswimmers have been investigated in condi- 
tions of externally imposed flow. Palacci et al. reported upstream motion of light-activated "surfers" consisting of hematite cubes embedded in a polymeric sphere ${ }^{[10]}$. Several papers have described a similar positive rheotactic response for bimetallic rods ${ }^{[11,12,13]}$. Interestingly, all of the microswimmers (both biological and artificial) discussed so far possess an anisotropy in shape, and their upstream response can be rationalized by appealing to a so-called "weather vane" mechanism ${ }^{[14,15]}$ : when swimming close to the surface, the swimmer body experiences an attraction to the substrate, and can therefore function as an "anchor" or pivot point. The tail extends away from the surface, into faster fluid flow. It therefore experiences a downstream viscous drag due to the flow, and the resulting torque aligns the swimmer upstream.

The response of spherical microswimmers, which have no distinction between major and minor axes like a weather vane, in an imposed flow is an intriguing question. Recently, two individual studies reported oscillatory positive rheotaxis for isotropic active droplets ${ }^{[16,17]}$. The rheotactic behavior was attributed to flow-induced distortions of the solute field ${ }^{[16]}$ and hydrodynamic interactions with the four walls of a confining Hele-Shaw microchannel ${ }^{[17]}$. Earlier, a theoretical study of Zöttl and Stark had predicted oscillatory positive rheotaxis of a spherical microswimmer exposed to Poiseuille flow in a cylindrical tube, another highly confined space ${ }^{[18]}$. A theoretical study from Uspal et al. predicted steady positive rheotaxis for spherical swimmers near a planar surface under certain conditions concerning the self-propulsion mechanism and flow speed ${ }^{[19]}$.

Notably, the study from Uspal et al., in contrast with the other studies, explicitly considered Janus particles, a paradigmatic example of a synthetic spherical swimmer. Janus particles are colloids with two distinctly different properties on the hemispheres: here, one inert and one catalytic half. The catalytic part degrades fuel molecules, leading to variations in the concentrations of the various molecules participating in the reaction and, for reactions involving charged 
intermediates, variations in the electrostatic potential. These self-created chemical and electrical gradients cause a flow in close vicinity of the surface, i.e., the so-called phoretic slip. For a concentration gradient, the diffusiophoretic slip is directly proportional to a surface-tangential component of the concentration gradient and a material-dependent parameter called the phoretic mobility ${ }^{[20]}$. The phoretic mobility encodes the molecular-scale interactions between the various molecules in solution and the particle surface. This resulting direction of the slip flow depends on the resultant effective interaction between material and solutes, and is described to be "difficult to predict a priori and depends on the details of the chemical reaction and species" by Popescu et al. ${ }^{[21]}$. The phoretic mobility can vary over the surface of the particle, e.g., between the catalytic and inert sides. Similarly, the electrophoretic slip is proportional to the tangential component of the electric field, with the coefficient of proportionality including the zeta potential. An on-going discussion in the literature concerns the relative importance of neutral self-diffusiophoretic, ionic self-diffusiophoretic, and self-electrophoretic mechanisms in Janus particle propulsion ${ }^{[22,23,24]}$.

Regardless of whether the slip is diffusiophoretic or electrophoretic in origin, the variation of the slip over the surface of the particle determines whether the particle has a hydrodynamic "pusher" or "puller" character. If the slip is strongest on the leading "face" of the particle, it is a puller; if it is strongest on the trailing face, it is a pusher. (Intuitively, one might think that the slip should be localized to the catalytic side of the particle and strongest there, but the slip on the inert side can be significant if the inert material has a large phoretic mobility.) The pusher or puller character of a microswimmer has a profound impact on how it interacts with its environment hydrodynamically, i.e., through self-generated fluid flows ${ }^{[25]}$. Thus, knowledge of the pusher/puller character of various Janus particles, although elusive, would be useful for designing various applications. Significantly, the study of Uspal et al. had predicted positive rheotaxis for puller-type Janus particles. 
Among spherical microswimmers, $\mathrm{Pt}$ coated insulator spheres (typically $\mathrm{SiO}_{2}$ or polystyrene), remains the most investigated system ${ }^{[26]}$. The $\mathrm{Pt}$ half catalyzes the decomposition of $\mathrm{H}_{2} \mathrm{O}_{2}$ fuel and the swimmer is known to move away from the metal cap. Various pieces of evidence suggest that these "inert-forward" particles are pushers. For instance, recently Campbell et al. have experimentally characterized the hydrodynamic flow field around these $\mathrm{Pt} @ \mathrm{SiO}_{2}$ microswimmers using particle tracking velocimetry and found them to resemble pushers ${ }^{[27]}$. Brown et al. studied the speed variations of these microswimmers in two-dimensional colloidal crystals and inferred a pusher-type hydrodynamic interaction with the crystal ${ }^{[28]}$. Katuri et al. conducted experiments on spherically symmetric microswimmers in an imposed flow, taking $\mathrm{Pt} @ \mathrm{SiO}_{2}$ as model system ${ }^{[29]}$. Quite counter-intuitively, they found that the balancing of torque due to flow and the torque due to activity resulted in a cross-stream migration for these motors, which was confirmed in a subsequent study ${ }^{[30]}$. This cross-stream behavior was also recovered in a boundary element model of a self-diffusiophoretic particle for a certain choice of parameters ${ }^{[29]}$. Notably, these parameters corresponded to the particle being a pusher. Experimental reports of synthetic puller-type microswimmers are comparatively scarce. Brosseau et al. report being able to control the pusher/puller character of a bimetallic rod by changing the lengths of the two metal segments ${ }^{[13]}$.

In this manuscript, we introduce $\mathrm{Cu} @ \mathrm{SiO}_{2}$ microswimmers, hypothesizing that these capforward spherical swimmers are self-electrophoretic pullers. Flow field calculations based on a thin-layer approximation and experimentally determined surface properties support this conjecture. As noted above, theory predicts that pullers exhibit rheotaxis in externally imposed shear flow. We indeed find that these swimmers spontaneously align with the flow and migrate upstream for a wide range of external flow speeds. Additionally, we demonstrate that using a simple squirmer model for a puller configuration, we can reproduce all observed behaviours. Thus, our study sparks understanding how different combinations of materials can be chosen to 
impart a "pusher" or "puller" character to a Janus particle. We characterize the rheotactic behaviour for this novel puller type microswimmer in microfluidic experiments, and find at large flow speeds an unexpected "jumping" phenomenon in which the particle axis is spontaneously attracted to the shear gradient plane, as with steady rheotaxis, but the particle continuously rolls with respect to the vorticity axis of the flow. Consequently, the particle exhibits oscillations in speed and height.

\section{Results and Discussion}
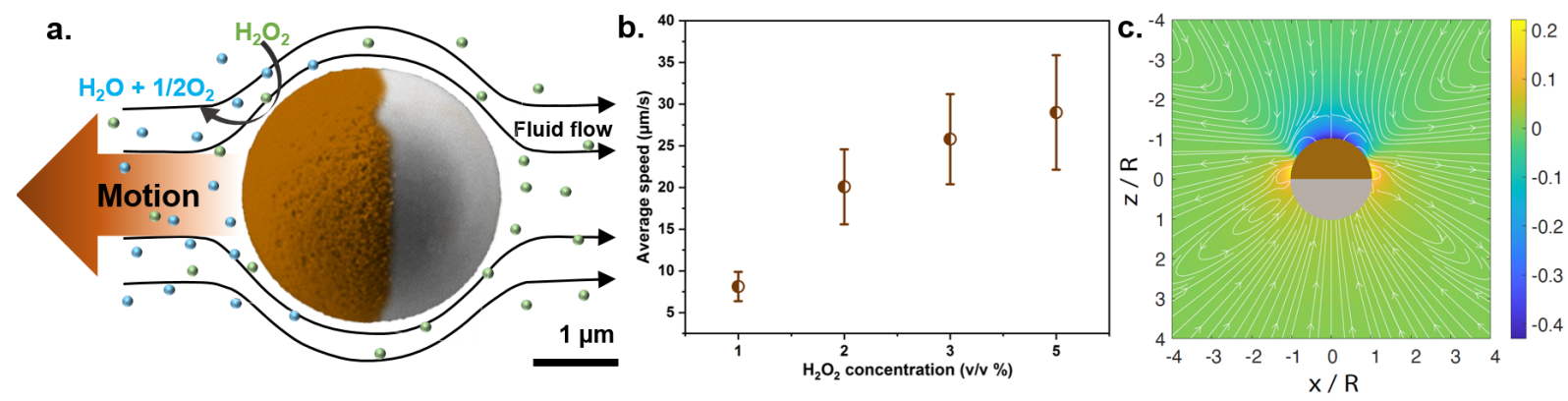

Figure 1: (a) Scanning Electron microscopy (SEM) image of a $\mathrm{Cu} @ \mathrm{SiO}_{2}$ Janus with illustrative propulsion mechanism in $\mathrm{H}_{2} \mathrm{O}_{2}$ solution. (b) Average speed of $\mathrm{Cu} @ \mathrm{SiO}_{2}$ micromotors in different hydrogen peroxide concentrations. (c) Electrostatic potential $\phi / \phi_{0}$ (background color) and streamlines for a self-electrophoretic $\mathrm{Cu} @ \mathrm{SiO}_{2}$ particle, according to the model presented in Methods. The streamlines are directed away from the equator and toward the poles, revealing the puller character of the Janus particle.

We begin by studying the motion characteristics of $\mathrm{Cu} @ \mathrm{SiO}_{2}$ Janus particles in absence of any external flows (Video $\mathrm{S} 1$ ). When suspended in $\mathrm{H}_{2} \mathrm{O}_{2}$ solution, the $\mathrm{Cu}$ hemisphere catalyzes decomposition of $\mathrm{H}_{2} \mathrm{O}_{2}$, leading to an asymmetric distribution of reaction products. The anisotropic presence of solutes in the vicinity of the particle surface leads to the formation of a surface flow, leading to directed motion (Fig.1a) and the average speed increased with increasing peroxide concentration (Fig.1b). Based on previous work, we hypothesize that an electrophoretic mechanism is responsible for self-propulsion ${ }^{[31]}$. 
In particular, the polar and near-equatorial regions of the particle are considered to act as cathode and anode, respectively, owing to variations in the thickness of the copper layer. We compute the electrostatic potential and flow field for this model in the thin-layer approximation (see Methods). The puller-type character of the flow is clearly apparent in Fig.1c, as streamlines are directed towards the poles and away from the equator. In experiments, and similar to $\mathrm{Pt} @ \mathrm{SiO}_{2}$ particles ${ }^{[32]}$, the particles sediment to the vicinity of a bounding solid surface (substrate). Moreover, in the presence of chemical "fuel," the particles align their axes of symmetry to be nearly parallel to the plane of substrate. Previously, we had rationalized this alignment for $\mathrm{Pt} @ \mathrm{SiO}_{2}$ by the superposed interactions of the solute interactions with the substrate and by chemiosmotic flows, both of which tend to rotate the cap away from the substrate, and bottomheaviness and hydrodynamic interactions, which tend to rotate the cap towards the substrate. For the case of $\mathrm{Cu} @ \mathrm{SiO}_{2}$ we assume the solute-particle effective interactions to be (weakly) attractive due to the slight positive charge of the copper hemisphere in diluted peroxide, resulting in opposite directions of flows as well as motion in direction of the metal cap. Still, it is remarkable that, despite different densities (weak bottom-heaviness), forces and torques again balance in a swimming orientation with their symmetry axis parallel to the substrate (compare also Ref. 33). 


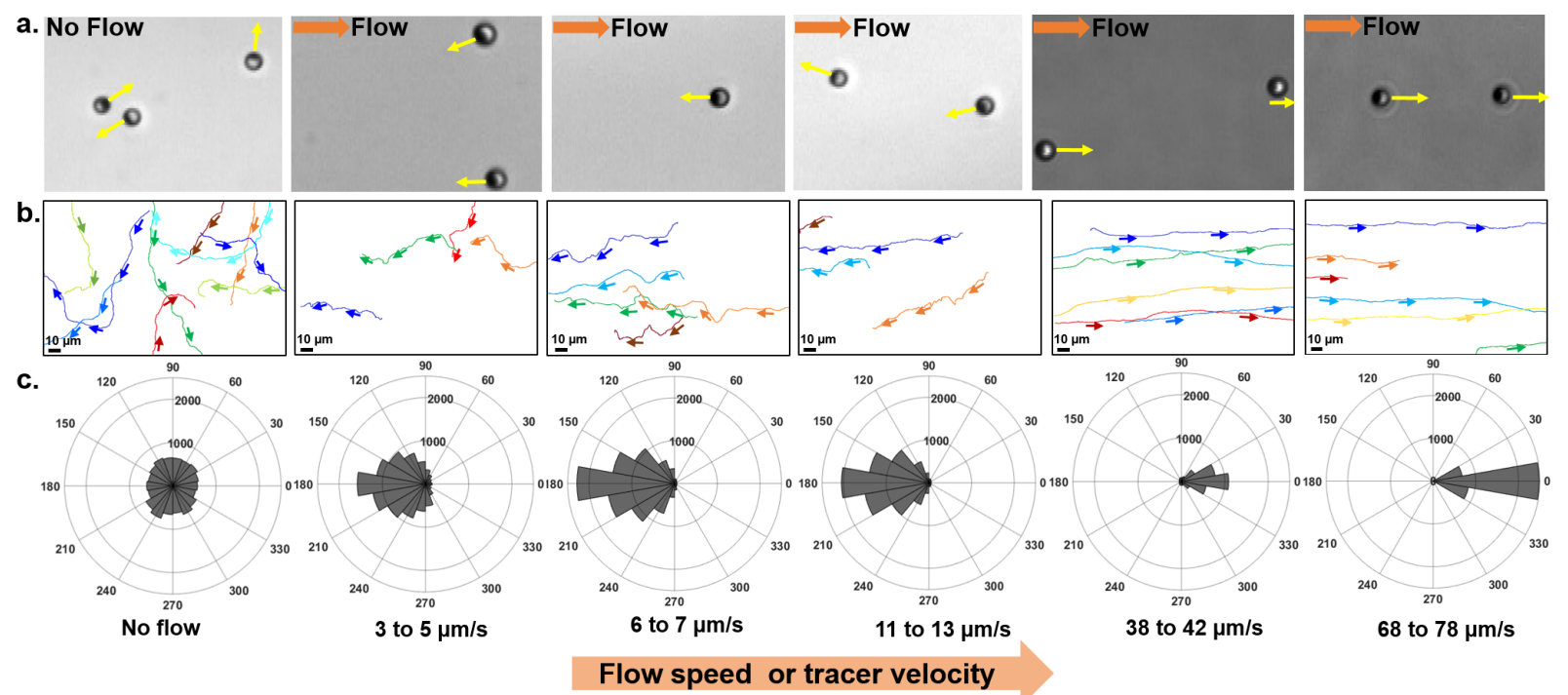

Figure 2: Behaviour of $\mathrm{Cu} @ \mathrm{SiO}_{2}$ particles in flow: (a) Optical micrograph capturing the orientation of Janus particles in no flow and in imposed flow conditions at different flow speeds. The flow speeds are indicated in the bottom panel. The orange arrows indicate the direction of flow and the yellow arrow indicate the direction of motion direction. (b) Trajectories of motion and (c) Rose plots capturing an overview of motion direction of $\mathrm{Cu} @ \mathrm{SiO}_{2}$ particles in different flow conditions. The values associated with different radii in the Rose plots indicate the number of time frames and the black length being proportional to the values in that direction.

To evaluate the behaviour in external flow, we start flowing a solution of diluted $\mathrm{H}_{2} \mathrm{O}_{2}$ through a glass capillary using a pressure pump (Video S2). The active Janus particles orient in the direction parallel to the flow and begin to move upstream. To characterize the swimming behaviour of these $\mathrm{Cu} @ \mathrm{SiO}_{2}$ Janus particles, we track their swimming directions in different applied flow rates and plot them in Fig.2. Passive $\mathrm{SiO}_{2}$ colloids were used as tracers to determine the flow speed in the vicinity of the active particles. In no-flow conditions the speed values for these particles are found be $\approx 22 \mu \mathrm{m} / \mathrm{s}$, so no detectable influence of the closed capillary is noted. As we begin to impose flow, the particles start to move against the direction of flow. For low flow speeds $(3-5 \mu \mathrm{m} / \mathrm{s})$, the previously described upstream swimming is clearly evident, but increasing the flow speed results in a better alignment of the active particles against the direction of flow. At even higher flow speeds $(38-42$ and $68-78 \mu \mathrm{m} / \mathrm{s})$, the particles are no longer able 
to swim against the flow. Their direction of motion is flipped by 180 degrees, but the particles still maintain their upstream orientation (i.e., catalytic cap oriented towards the source of flow.) These behaviours were confirmed by plotting the angle probability distribution of a population of $\mathrm{Cu} @ \mathrm{SiO}_{2}$ Janus particles (see Fig.2c). The motion direction of each particle at every frame was plotted to determine the probability distribution (see Scheme 1 in methods section).
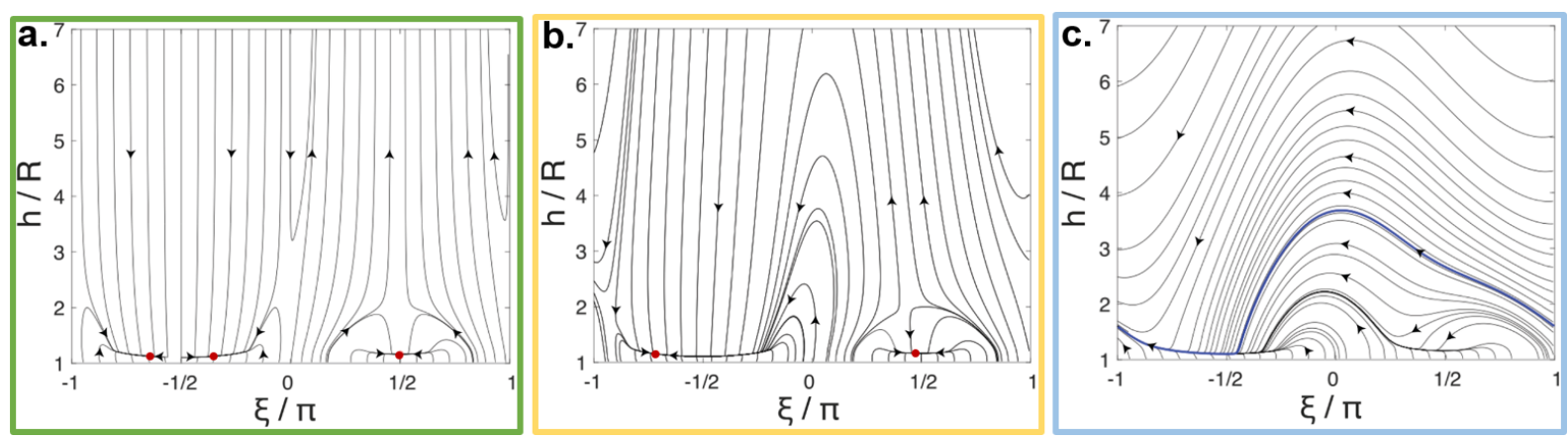

Figure 3: Simplified phase portraits for in-plane dynamics of a particle with $\beta=7, G=0.3$, $T=0, E=100$, and (a) $S=0$ (no flow) (b) $S=0.1$, where good agreement between the observed and predicted behaviour can be observed (c) Phase portrait for $S=1$, the absence of attractor entails that the particle continuously rotates. Red dots indicate attractors.

In order to understand these observations, we use a simple theoretical model of a squirmer exposed to shear flow near a planar wall. By treating the particle as an effective squirmer, we coarse-grain the detailed interactions between the particle and the wall (e.g., chemical, electrostatic, hydrodynamic, and electroosmotic), which depend on the mechanism of propulsion. ${ }^{[34]}$ However, the results of self-electrophoretic model for $\mathrm{Cu} @ \mathrm{SiO}_{2}$ suggest treating the particle as a puller. Overall, as detailed below, we find that puller-type squirmers can exhibit the full range of behavior seen in the experiments. Pusher-type squirmers, in contrast, exhibit cross-stream alignment at high flow rates, i.e., the orientation of the particle is unstable against perturbations of the polar axis out of the shear plane ${ }^{[19,29]}$.

In the Methods section, we present our model in greater detail. It is parameterized by $\beta, G$, $T, S$, and $E$, which respectively represent the pusher/puller character of the particle, the particle 
bottom-heaviness, the flow strength, and the strength of electrostatic repulsion from the wall. The instantaneous configuration of the particle is specified by the orientation vector $\hat{p}$ and the height $h / R$ of the particle above the wall, where $R$ is the particle radius. A planar substrate is located at $z=0$, and the flow is in the $y$-direction.

First, we consider the case when $p_{x}=0$, i.e., the particle orientation $\hat{p}$ is in the shear plane. By symmetry, if $\hat{p}$ is in the shear plane, it will remain there for all time (in the absence of out-ofplane fluctuations.) In Fig.3, we show phase portraits for a squirmer with $\beta=7$ (i.e., a puller), $G=0.3, T=0$, and $E=100$ for $S=0$ (no ambient flow), $S=0.1$, and $S=1$. The red dots indicate stable fixed points for the in-plane dynamics, i.e., attractors. Fig.3a shows the case of no flow, i.e., $S=0$. Since there is no ambient flow to break the symmetry with respect to $\hat{\mathbf{e}}_{z}$ (Scheme 2a in the methods section), there is a mirror symmetry between regions I and II, as well as between regions III and IV (where the regions are defined as follows: $-\pi<\xi<\pi / 2$ is region $\mathrm{I},-\pi / 2<\xi<0$ is region II, $0<\xi<\pi / 2$ is region III, and $-\pi / 2<\xi<\pi$ is region IV; see also Scheme $2 b$ in the Methods section). Thus, for instance, for the attractor ("sliding state") in region II, for which the cap is oriented towards the wall and in the positive $y$-direction, there is a corresponding attractor in region I, for which the cap is oriented towards the wall and in the negative $y$-direction. These two attractors are of particular interest, because they capture the experimental observation that, in the absence of flow, particles tend to move with their axes of symmetry approximately parallel to the wall. There is another attractor at $\xi=\pi / 2$, i.e., on the boundary between regions III and IV. A particle in this state is "hovering" with its cap oriented away from the wall. This hovering state is induced by the repulsive potential between the particle and wall. However, the basin of attraction of this state is limited.

Now we consider the case of low flow speed $(S=0.1)$ in Fig.3b. There is still an attractor in region I. This attractor corresponds to upstream rheotaxis, i.e., the cap is oriented upstream and towards the wall. There is also an attractor in region III, but this state is of less interest, as 
the cap is oriented away from the wall and downstream.

In Fig.3c, we show the case of high flow speed $(S=1)$. It is remarkable that there are no longer any attractors, contrary to what one might expect from the rheotactic behavior at low flow rates. However, trajectories cross the phase space from high $\xi$ to low $\xi$ (the phase space is periodic at $\xi=-\pi$ and $\xi=\pi$.) In region I, many trajectories become arbitrarily close to "slow manifold" near the wall (without actually overlapping.) Thus, many trajectories tend to evolve towards the solid curve. The periodic behavior of the solid curve corresponds to a particle rolling downstream with its orientation in the shear plane. Interestingly, over one period of this oscillation, a particle will be aligned both upstream and downstream. Thus, the particle is swimming both with and against the flow, and the speed of the particle oscillates accordingly. Moreover, the height of the particle oscillates significantly in this "jumping" regime. 

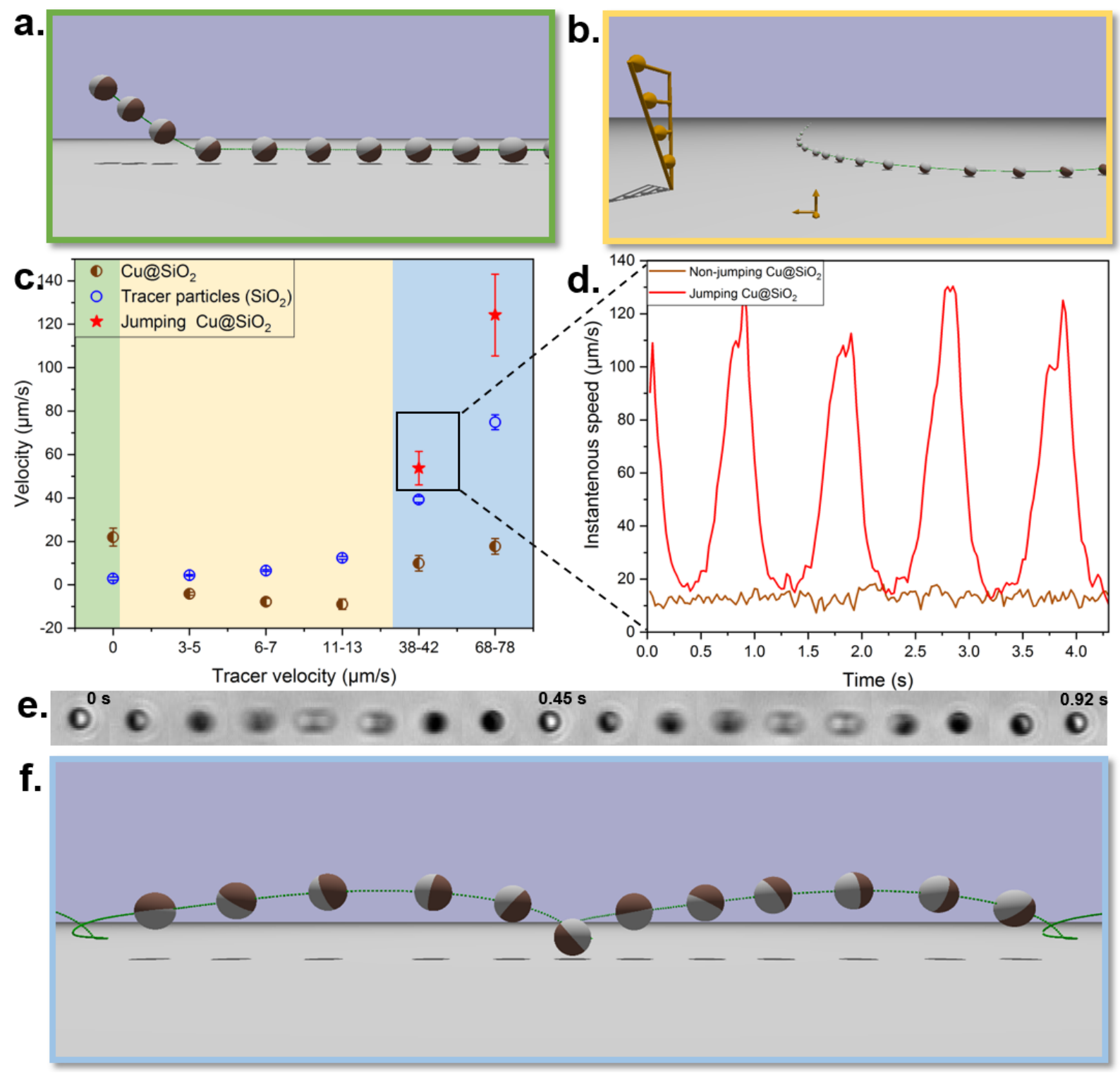

Figure 4: Three-dimensional view of particle trajectory (a) in no flow conditions, (b) upstream rheotaxis. (c) Velocity of $\mathrm{Cu} @ \mathrm{SiO}_{2}$ particles in different no flow/ flow conditions. (d) Instantaneous speed of a "jumping" and "non-jumping" $\mathrm{Cu} @ \mathrm{SiO}_{2}$ particle in a very high flow speed (38-42 $\mu \mathrm{m} / \mathrm{s})$. (e) Time lapse optical images depicting two cycles of jumping behaviour. The particle goes out of focus in the jumping time. (f) Three-dimensional view of a jumping trajectory.

This novel theoretical prediction inspired us to closely observe the experimental outcome at high flow speeds. Indeed, besides the steady particles orienting for rheotactic behaviour but 
being dragged by the flow, we observed particles rolling as they were swept downstream by the flow (Video S3). Moreover, these particles periodically detached from and re-approached the surface, as evidenced by periodic defocusing and refocusing of the particles in the microscope field of view (Fig.4e). A three-dimensional view of jumping is shown in Fig.4f. Quantitatively, jumping was captured by plotting the instantaneous speed of an individual jumping particle (Fig.4d). The periodic behaviour with the peaking speed values is clearly evident. For the depicted particle, the wavelength is just below $1 \mathrm{~s}$ and seems stable, despite the fact that long term analysis is restricted by the field of view. In addition to jumping particles, we also noticed that a set of $\mathrm{Cu} @ \mathrm{SiO}_{2}$ particles remained close to the surface throughout the period of study and aligned their Janus axis of symmetry in the shear plane ${ }^{[30]}$ (Fig.4c). The presence of two different populations may be due to variation in particle self-propulsion speed. 

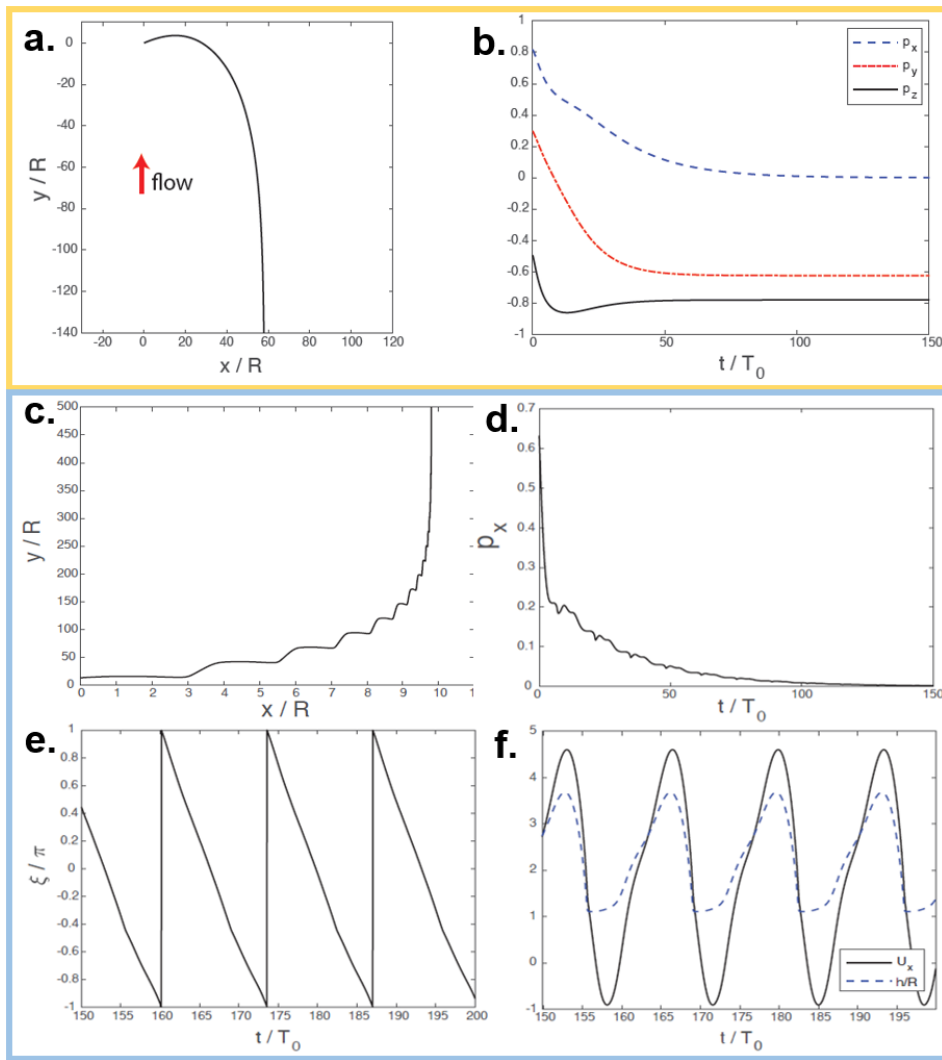

Figure 5: Simulation data for a particle that exhibits upstream rheotaxis. The flow strength $S=$ 0.1 , and all other parameters are the same as in Fig.3. The initial height is $\mathrm{h} / \mathrm{R}=1.1$, and initial orientation is $\theta=120^{\circ}$ and $\phi=20^{\circ}$. (a) Projection of the particle trajectory in the xy-plane. The particle is initially swept downstream before it achieves a rheotactic orientation and swims upstream. (b) Time evolution of the three components of $\hat{p}$. The out-of-plane component $\mathrm{p}_{x}$ decays to zero. The other two components are attracted to the angle $\xi$ of region I attractor in Fig.3b. Simulation data for a particle with jumping dynamics. The flow strength $S=1$, and all other parameters are the same as in Fig.3. The initial height is $\mathrm{h} / \mathrm{R}=1.1$, and initial orientation is $\theta=40^{\circ}$ and $\phi=10^{\circ}$. (c) Projection of the trajectory in the xy-plane. (d) Time evolution of the out-of-plane component $p_{x}$. (e) Time evolution of the angle $\xi$ when the particle is "jumping" with $\mathrm{p}_{x} \approx 0$. (f) Time evolution of the streamwise velocity $\mathrm{U}_{y}$ and height $\mathrm{h} / \mathrm{R}$ in the jumping state.

The preceding theoretical analysis recovered the full range of behaviors observed in experiments. However, since it assumed $p_{x}=0$, we have not yet shown that these behaviors occur for an arbitrary initial orientation of the particle, i.e., that the particles spontaneously align in the shear plane. In fact, this issue provides a crucial distinction between pullers and pushers: while 
pullers spontaneously align in the shear plane, pushers are unstable against rotation of their orientation out of the shear plane. Instead, pushers are spontaneously attracted to a cross-stream migration state. ${ }^{[19,29]}$

To investigate the stability against out-of-plane rotation, we conduct further theoretical analysis considering the case in which the particle orientation $\hat{p}$ has a component outside of the shear plane $\left(p_{x} \neq 0\right)$. In a previous work (Ref. 19), we analyzed the linear stability of the fixed points for in-plane dynamics against perturbations of $\hat{p}$ out of the shear plane. We found that these fixed points can be stable against out-of-plane perturbations when they are located in regions I and III. Therefore, the upstream rheotactic attractor in Fig.3b should be robust against perturbations of the orientation out of the shear plane. Fig.5a, 5b shows the trajectory of a particle with an initial height $h / R=1.1$ and initial orientation of $\theta=120^{\circ}$ and $\phi=20^{\circ}$ for $S=0.1$, and other parameters as given in the caption of Fig.3. The $x$-component of the orientation decays to zero, while $p_{y}, p_{z}$, and $h / R$ evolve towards steady values, corresponding to the location of the region I attractor in Fig.3b.

We note that the presence of an attractor in Region I in Fig. 3b is consistent with of an analysis of the hydrodynamic interaction of a squirmer and a wall using a far-field approximation. In this analysis, near-surface swimming activity tends to rotate the squirmer as follows: ${ }^{[35]}$

$$
\frac{\dot{\xi}^{(s)} R}{U_{\mathrm{fs}}}=\frac{3}{16}\left(\frac{R}{h}\right)^{3}\left[\frac{3}{4} \beta \sin 2 \xi+\frac{R}{h} \cos \xi\right] .
$$

Since the contribution of shear flow is $\dot{\xi}^{(f)}<0$, the term in brackets in Eq. 1 must be positive for flow and hydrodynamic interactions to balance each other and produce a steady state. (We recall that rotation by bottom-heaviness is negligible for our particles.) For $\xi$ in region I, $\sin 2 \xi$ is positive and $\cos \xi$ is negative. Accordingly, we need $\beta>0$, i.e., the squirmer must be a puller. Stated another way, pullers tend to rotate their swimming direction to point towards solid bounding surfaces; for a squirmer oriented upstream and towards the wall, this tendency 
can counteract rotation by the flow. (Interestingly, this tendency can change sign for a puller very close to the wall, i.e., in the lubrication regime; ${ }^{[35]}$ however, for the particle size and Debye length considered here, the particle is in the far-field regime.) Additionally, this far-field analysis also shows that hydrodynamic interactions cannot produce upstream rheotaxis for pushers, for which $\beta<0$.

For the situation corresponding to Fig.3c, there are no attractors for in-plane dynamics. Thus, the linear stability analysis in Ref. 19, which was developed for attractors, is not applicable here. However, we find that the squirmer spontaneous evolves towards in-plane "jumping." In other words, an initial out-of-plane component $p_{x}$ of the orientation decays over time to zero, as shown in Fig.5d. Therefore, the projection of the trajectory in the $x y$-plane asymptotes to a straight line, as seen in Fig.5c. From the time evolution of $p_{x}$, it is apparent that $p_{x} \approx 0$ for $T / T_{0}>150$. Thus, in Fig.5e, we show the in-plane angle $\xi$. Clearly, the particle is periodically rotating in the direction (clockwise) expected from the shear profile (see Scheme 2). In Fig.5f, we recover the "jumping" phenomenon, i.e., oscillation of the particle speed in the $y$-direction. In this case, the particle actually shows intermittent upstream swimming $\left(U_{y}<0\right)$ when it is aligned against the flow, but the net motion is in the $y$-direction.

We can get a sense of why jumping is stable against out-of-plane rotations by considering a small perturbation $\Delta p_{x} \neq 0$. This perturbation will slowly decay to zero (see Fig.5d); the vertical motion and "rolling" will be much faster. Accordingly, we can think about averaging $\dot{p}_{x}$ over the solid curve in Fig.3c to obtain a period-averaged $\left\langle\dot{p}_{x}\right\rangle$. For the solid curve in Fig.3c, the particle is closest to the wall when it is oriented upstream and towards the wall (Region I). Moreover, in Fig.5e, the particle spends appreciable time - roughly a third of each period oriented upstream and towards the wall. Because of its small height above the wall, the particle will have strong hydrodynamic interactions with the wall, and this part of the periodic motion will dominate $\left\langle\dot{p_{x}}\right\rangle$. It was shown in Ref. 19, in the context of steady rheotaxis, that a puller 
oriented upstream and towards the wall will be stable against out-of-plane rotations (see Eq. 8 in that work.) Thus, during this part of the periodic motion, $\Delta p_{x}$ will decay. The net result over one period is that $\Delta p_{x}$ will decay.

\section{Conclusions}

To conclude, we report the dynamics of spherically symmetrical $\mathrm{Cu} @ \mathrm{SiO}_{2}$ Janus colloids. Unlike the well studied $\mathrm{Pt} @ \mathrm{SiO}_{2}$ system, the $\mathrm{Cu}$ coated $\mathrm{SiO}_{2}$ particles demonstrate a cap-forward motion, when suspended in $\mathrm{H}_{2} \mathrm{O}_{2}$ solution while still maintaining their Janus normal parallel to the motion vector. Based on previous studies on a similar system, we conjecture a selfelectrophoretic model responsible for their propulsion. Theoretical flow field calculations using this model in the thin-layer approximation result into a puller-type behaviour for these active colloids. Interestingly, earlier theoretical results have predicted an upstream directional response for puller-type Janus colloids in conditions of imposed flow, implicating interplay of hydrodynamic interaction, shear flow near a surface. When looking at the response of $\mathrm{Cu} @ \mathrm{SiO}_{2}$ swimmers in the conditions of an externally imposed flow in a closed capillary, we capture their steady upstream migration with striking similarities to theoretical predictions. We recover all the experimental observations using a puller squirmer model and in addition, the model predicts a unique "jumping" phenomenon at high flow rates. This novel behaviour is captured when performing the experiments at comparatively higher flow rates, whereby, the particle periodically detaches and re-approaches the surface. In this process, the particle continuously rolls out of plane besides being dragged downstream.

While earlier studies of flow response on well understood pusher-type Janus colloids have reported cross-stream behaviour, this manuscript presents rare puller type Janus microswimmers demonstrating and investigates their upstream swimming. Based on flow field calculations and particularly their response in external flow we conjecture a puller-type configuration for 
$\mathrm{Cu} @ \mathrm{SiO}_{2}$ Janus colloids. Our findings also suggest, that in the future the response of an active particle to an external flow can be used to infer a pusher or puller character.

\section{Materials and Methods}

\section{Preparation of $\mathrm{Cu} @ \mathrm{SiO}_{2}$ Janus colloids}

Janus colloids were obtained by drop casting a suspension of $3 \mu \mathrm{m}$ diameter $\mathrm{SiO}_{2}(\mathrm{Sigma}$ Aldrich) particles in ethanol, on a plasma clean glass slide followed by slow evaporation of the solvent. Subsequently, a $30 \mathrm{~nm}$ thin layer of $\mathrm{Cu}$ was thermally deposited on the monolayer. The $\mathrm{Cu} @ \mathrm{SiO}_{2}$ particles were released in deionized (DI) water by applying short ultrasonic pulses.

\section{Behaviour of $\mathrm{Cu} @ \mathrm{SiO}_{2}$ particles in flow}

Square glass capillaries ( $1 \mathrm{~mm} * 1 \mathrm{~mm}, 0.2 \mathrm{~mm}$ wall thickness, $100 \mathrm{~mm}$ length, Vitrocom) used in these experiments were pre-soaked in $30 \% \mathrm{w} / \mathrm{w} \mathrm{H}_{2} \mathrm{O}_{2}$ overnight (to oxidize any organic substance present on the surface) and were subsequently flushed several times with DI water and dried. An aqueous suspension of $3 \mu \mathrm{m} \mathrm{Cu} @ \mathrm{SiO}_{2}$ containing $3 \mu \mathrm{m} \mathrm{SiO}$ particles as tracers, is introduced into the capillary before mounting it onto an in-house 3D printed microscope stage (see SI Fig.S1). The particles are allowed to sediment at the substrate before any external flow is applied. Subsequently, the capillary is connected to a microfluidic pressure pump (Flow EZ Fluigent) via polyethylene tubings. We begin by flowing $2.5 \mathrm{w} / \mathrm{w} \% \mathrm{H}_{2} \mathrm{O}_{2}$ in the capillary by manually controlling the pressure. The flow speed was determined using the tracer particles. The exact same procedure as above was done for no-flow experiments, except, the square capillary was not connected to the tubings. Here, $2.5 \mathrm{w} / \mathrm{w} \% \mathrm{H}_{2} \mathrm{O}_{2}$ was introduced into the capillary with the help of a pipette tip. The motion was recorded at a frame rate of $40 \mathrm{fps}$ using $50 \mathrm{X}$ magnification. 


\section{Motion studies of $\mathrm{Cu} @ \mathrm{SiO}_{2}$ particles in different hydrogen peroxide solu- tions}

On a plasma cleaned glass slide (pre-cleaned with acetone and ethanol), $1 \mu \mathrm{L}$ of an aqueous suspension of $3 \mu \mathrm{m} \mathrm{Cu} @ \mathrm{SiO}_{2}$ particles was placed. After, the particles sediment, $20 \mu \mathrm{L}$ of diluted $\mathrm{H}_{2} \mathrm{O}_{2}$ solution was added. The motion of the particles was recorded at 40 frames per second rate using 50X magnification.

\section{Video recording}

The motion was recorded on an attached Zeiss camera (Axiocam 702 Mono) to a Carl Zeiss inverted microscope.

\section{Motion Analysis}

After appropriate thresholding the videos were analyzed using Trackmate plugin in ImageJ and the speed was obtained as an average of a minimum of 15 particles. For the jumping $\mathrm{Cu} @ \mathrm{SiO}_{2}$, the speed was calculated as the time taken by the particle to cross the view frame (expecting the particle move in a straight line).

\section{Direction Analysis}

To obtain the motion direction of $\mathrm{Cu} @ \mathrm{SiO}_{2}$ particles, we used MATLAB to coordinate every position of moving particles and calculate the instantaneous direction changes of them in each time frame. To elaborate, when a particle moved from frame $t_{1}$ to $t_{3}$, three angles $\left(\theta_{1}, \theta_{2}, \theta_{3}\right)$ between trajectories and horizon axis were calculated and recorded accordingly. 


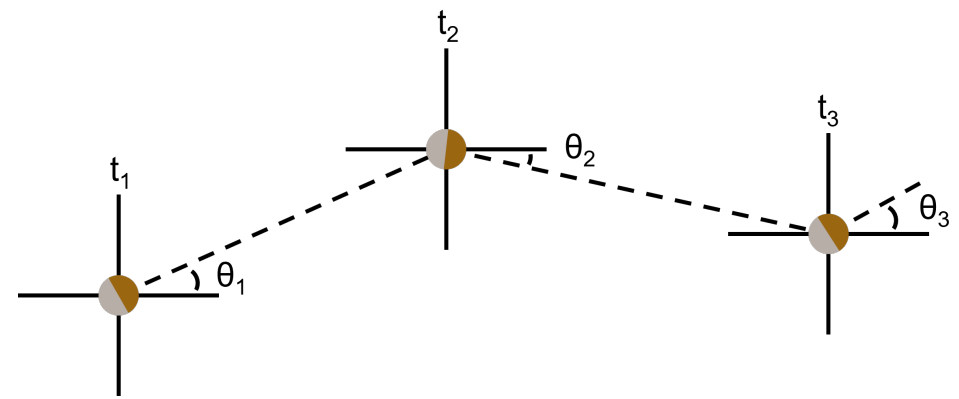

Scheme 1: Instantaneous direction changes of a moving particle

\section{Self-electrophoretic model}

The self-electrophoretic propulsion mechanism of the particle is modeled as follows. It has been hypothesized that the region of the catalytic surface near the "pole," which is thicker than the region near the "equator" due to the deposition process, acts as the cathode and produces $\mathrm{H}+$ ions. The region near the equator acts as the anode and consumes $\mathrm{H}+$ ions. We assume that the distribution of $\mathrm{H}+$ ions is quasi-steady, that the Debye layer is thin compared with the particle radius, and that the production and consumption of ions can be modeled with constant and uniform fluxes on the particle surface. Additionally, we consider a particle in free space (i.e., far from any bounding surfaces) with its center at the origin, and with its propulsion axis aligned with the $z$-axis.

Accordingly, the electrostatic potential $\phi$ in solution is described by the Laplace equation $\nabla^{2} \phi=0$. As boundary conditions, we specify $\phi(|\mathbf{x}| \rightarrow \infty)=0$, i.e., the potential decays to zero far from the particle, and $\hat{n} \cdot \nabla \phi=J k_{B} T g(z) / 2 e D n_{0}$ on the particle surface. ${ }^{[36,31]}$ Here, $J$ is the flux of ions, $k_{B} T$ is the thermal energy, $e$ is the elementary electronic charge, $D$ is the diffusion coefficent of $H+$ ions, and $n_{0}$ is the bulk concentration (number density) of $H+$ ions. The surface normal $\hat{n}$ is directed into the fluid. The function

$$
g(z)=\left\{\begin{array}{ll}
0 & z \leq 0 \\
-1 & 0<z \leq R / 2 \\
1 & R / 2<z \leq R
\end{array}\right\}
$$


where $R$ is the particle radius, accounts for the cathode and anode character of the particle surface. Note that for this $g(z)$ function, the two regions have equal area; therefore, the rates of ion production and consumption are balanced. We nondimensionalize the governing equation for $\phi$ by choosing $\phi_{0} \equiv J k_{B} T R / 2 e D n_{0}$ as a characteristic voltage and $R$ as a characteristic length scale. Following Ref. 31, we use $D=9.3 \times 10^{-9} \mathrm{~m}^{2} / \mathrm{s}$ as the diffusivity of $\mathrm{H}+$ ions, $n_{0}=2.24 \times 10^{-3} \mathrm{~mol} / \mathrm{m}^{3}$ as the bulk $\mathrm{H}+$ concentration for a solution saturated with atmospheric $\mathrm{CO}_{2}, R=1.5 \mu \mathrm{m}$, and $J=10^{-5} \mathrm{~mol} / \mathrm{m}^{2} \mathrm{~s}$, we obtain a characteristic voltage of $9 \mathrm{mV}$.

Concerning the particle and fluid velocity, we assume incompressible low Reynolds number flow. The flow is therefore governed by the Stokes equation $-\nabla p+\mu \nabla^{2} \mathbf{u}=0$, where $\mathbf{u}(\mathbf{x})$ is the fluid velocity, $p(\mathbf{x})$ is the pressure, $\mu$ is the dynamic viscosity, and $\mathbf{x}$ is a position in the solution. The incompressibility condition gives $\nabla \cdot \mathbf{u}=0$. On the surface of the particle, the fluid velocity obeys the no-slip boundary condition $\mathbf{u}=\mathbf{U}+\boldsymbol{\Omega} \times\left(\mathbf{x}-\mathbf{x}_{p}\right)+\mathbf{v}_{s}\left(\mathbf{x}_{s}\right)$, where $\mathbf{x}_{p}$ is the position of the particle center, $\mathbf{x}_{s}$ is a point on the particle surface, and $\mathbf{U}$ and $\Omega$ are the unknown translational and angular velocities of the particle, respectively. (By axisymmetry, we expect $\Omega=0$.) The slip velocity $\mathbf{v}_{s}\left(\mathbf{x}_{s}\right)=-\frac{\epsilon_{r} \epsilon_{0} \zeta\left(\mathbf{x}_{s}\right)}{\mu} E_{\|}$, where $\epsilon_{0}$ is the permittivity of free space, $\epsilon_{r}$ is the relative permittivity of the solution, $\zeta\left(\mathbf{x}_{s}\right)$ is the (position-dependent) zeta potential, and $E_{\|}=-\nabla_{\|} \phi$ is the tangential component of the electric field. The velocity decays to zero far from the particle, i.e., $\mathbf{u}(|\mathbf{x}| \rightarrow \infty) \rightarrow 0$. Moreover, the particle is force-free and torque-free. Accordingly, the hydrodynamic force $\mathbf{F}_{H}=0$ and torque $\boldsymbol{\tau}_{H}=0$, where $\mathbf{F}_{H}=\int_{S} \boldsymbol{\sigma} \cdot \hat{\mathbf{n}} d S$ and $\boldsymbol{\tau}_{H}=\int_{S}\left(\mathbf{x}-\mathbf{x}_{p}\right) \times \boldsymbol{\sigma} \cdot \hat{\mathbf{n}} d S$. The integrals are taken over the particle surface $S$, and $\sigma=\mu\left(\nabla \mathbf{u}+\nabla \mathbf{u}^{T}\right)$ is the fluid stress tensor. The force-free and torque-free conditions close the system of equations for $\mathrm{U}$ and $\Omega$.

We nondimensionalize velocity by defining a characteristic velocity $U_{0}=\frac{\epsilon_{r} \epsilon_{0} \zeta_{\mathrm{cu}} \phi_{0}}{\mu R}$. The contrast between the copper and silica zeta potentials provides a dimensionless parameter $\tilde{\zeta}=$ $\zeta_{\mathrm{siO} 2} / \zeta_{\mathrm{Cu}}$. By solving the (nondimensionalized) model for $\tilde{\zeta}=-2.15$ (justification given be- 
low), we obtain a swimming velocity of $\mathbf{U}=U_{\mathrm{fs}} \hat{\mathbf{e}}_{z}$, where the free space swimming velocity is $U_{\mathrm{fs}} / U_{0}=0.33$.

We can obtain the swimming velocity in dimensional units by specifying $\zeta_{C u}=14 \mathrm{mV}$ and $\zeta_{\mathrm{SiO} 2}=-30 \mathrm{mV}$ (see SI), with $\epsilon_{r}=80$ and $\mu=10^{-4} \mathrm{~Pa}$ s for water. We thereby obtain a dimensional swimming speed of $U_{\mathrm{fs}}=19.8 \mu \mathrm{m} / \mathrm{s}$, which is comparable to the measured swimming speeds. (Note that the measurements are for a particle sliding along a wall, not a particle moving in free space.)

\section{Squirmer model}

We consider a spherical squirmer in the vicinity of a planar wall, located at $z=0$. The ambient flow is in the $y$-direction, and given by the flow profile $\mathbf{u}_{e x t}=\dot{\gamma} z \hat{e}_{y}$, where $\dot{\gamma}$ is the shear rate. The flow is governed by the Stokes equation and is incompressible.

On the surface of the squirmer, the fluid velocity obeys the no-slip boundary condition $\mathbf{u}=\mathbf{U}+\boldsymbol{\Omega} \times\left(\mathbf{x}-\mathbf{x}_{p}\right)+\mathbf{v}_{s}\left(\mathbf{x}_{s}\right)$, where $\mathbf{x}_{p}$ is the instantaneous position of the squirmer center, $\mathbf{x}_{s}$ is a point on the squirmer surface, and $\mathbf{U}$ and $\Omega$ are the unknown translational and angular velocities of the particle, respectively. On the stationary wall, the boundary condition is $\mathbf{u}=0$. Far from the particle, $\mathbf{u} \rightarrow \mathbf{u}_{e x t}$, where $\mathbf{u}_{e x t}=\dot{\gamma} z \mathbf{e}_{y}$ is the ambient flow profile. The slip velocity is written as a the sum of two squirming modes:

$$
\mathbf{v}_{s}\left(\mathbf{x}_{s}\right)=\left[B_{1} \sin \left(\theta_{p}\right)+B_{2} \sin \left(\theta_{p}\right) \cos \left(\theta_{p}\right)\right] \hat{\mathbf{e}}_{\theta_{p}},
$$

where $\theta_{p}$ is the polar angle in a frame in which the $\hat{\mathbf{e}}_{z}$ axis is aligned with the orientation $\hat{p}$ of the particle, and the unit vector $\hat{\mathbf{e}}_{\theta_{p}}$ is tangent to the particle surface. The quantities $B_{1}$ and $B_{2}$ are specified squirming mode amplitudes. The velocity of a squirmer in free space is $U_{\mathrm{fs}}=2 B_{1} / 3$. Accordingly, we nondimensionalize velocity using $U_{\mathrm{fs}}$ as a characteristic velocity, and define $\beta \equiv B_{2} / B_{1}$ as a dimensionless parameter. The sign of the parameter $\beta$ determines the pusher/puller character of the squirmer: $\beta>0$ for pullers and $\beta<0$ for pushers. 
Additionally, the particle is exposed to gravity (it is more dense than the solution) and a short-ranged repulsive force from the wall. We assume low particle Reynolds number, such that the forces on the particle sum to zero, i.e., $\mathbf{F}_{H}+\mathbf{F}_{G}+\mathbf{F}_{E S}=0$. Here $\mathbf{F}_{G}$ is the buoyant force on the particle; $\mathbf{F}_{H}=\int_{S} \boldsymbol{\sigma} \cdot \hat{\mathbf{n}} d S$ is the hydrodynamic force on the particle, where the integral is taken over the particle surface $S ; \boldsymbol{\sigma}=\mu\left(\nabla \mathbf{u}+\nabla \mathbf{u}^{T}\right)$ is the fluid stress tensor; and $\hat{\mathbf{n}}$ is the local normal on the surface of the particle, defined to point into the fluid. We assume that the repulsive force is of the form $\mathbf{F}_{E S}=F_{e s}(h / R) \hat{\mathbf{e}}_{z}$, with

$$
F_{e s}(h / R)=\frac{F_{0}^{e s} e^{-k R(h / R-1) / 2}}{\cosh (k R(h / R-1) / 2)},
$$

neglecting a possible difference in the interactions of the two "faces" of the particle with the surface. Here, $F_{0}^{e s}$ is a characteristic scale of the electrostatic force, and $k^{-1}$ is a characteristic length scale. Moreover, the torques on the particle balance: $\boldsymbol{\tau}_{H}+\boldsymbol{\tau}_{G}=0$, where $\boldsymbol{\tau}_{H}=$ $\int_{S}\left(\mathbf{x}-\mathbf{x}_{p}\right) \times \boldsymbol{\sigma} \cdot \hat{\mathbf{n}} d S$ is the hydrodynamic torque, and $\boldsymbol{\tau}_{G}$ accounts for the bottom-heaviness induced by the catalytic cap. As we will show below, $\tau_{G}$ is negligible. The force-balance and torque-balance equations close the system of equations for the unknown $\mathrm{U}$ and $\Omega$.

As a characteristic velocity, we use the free-space swimming velocity $U_{\mathrm{fs}}=2 B_{1} / 3$, and as a characteristic length, we use the particle radius $R$. We define the following dimensionless parameters. The pusher/puller character of the particle is parameterized by $\beta \equiv B_{2} / B_{1}$. The flow strength is parameterized by $S=\dot{\gamma} R / U_{\mathrm{fs}}$. The electrostatic force is parameterized by $E \equiv F_{0}^{e s} / 6 \pi \mu U_{\mathrm{fs}} R$. Sedimentation is parameterized by $G \equiv F_{G} / 6 \pi \mu R U_{\mathrm{fs}}$. Finally, bottomheaviness is parameterized by $T \equiv \tau_{G \text {, max }} / 6 \pi \mu R^{2} U_{\mathrm{fs}}$, where $\tau_{G \text {,max }}$ is the maximum torque from bottom-heaviness.

The effects of gravity are calculated using the "eggshell" model of Campbell and Ebbens. ${ }^{[37]}$ Using $\rho_{\mathrm{Cu}}=8900 \mathrm{~kg} / \mathrm{m}^{3}$ and $\rho_{\mathrm{SiO} 2}=2196 \mathrm{~kg} / \mathrm{m}^{3}$ for a film thickness of $t=30 \mathrm{~nm}$, we obtain $G \approx 0.3$. The bottom-heaviness parameter $T \approx 0.004$, and is hence negligible. Concerning 
electrostatic repulsion, we set $E=100$ and $k R=0.025$, which is sufficient to keep the particle out of contact with the wall. In dimensional units, $k^{-1}=30 \mathrm{~nm}$, consistent with an estimated Debye length of $44 \mathrm{~nm}$.

Our approach to particle dynamics exploits the symmetry of the problem. First, translational symmetry in the $x$-and $y$-directions implies that the configuration of the particle is specified by the height $h=z_{p}$ of the particle center above the wall and the orientation $\hat{p}$. Since $|\hat{p}|=1$, this configuration space is three-dimensional. The orientation vector $\hat{p}$ can also be specified by the angles $\theta$ and $\phi$, i.e., by the orientation of $\hat{p}$ in a spherical coordinate system. The height and orientation evolve according to $\dot{h}=U_{z}$ and $\dot{\hat{p}}=\Omega \times \hat{p}$.

We also exploit the the following implication of symmetry: if the particle orientation vector $\hat{p}$ is within the shear plane, i.e., the $y z$-plane, it must remain there for all time (within the deterministic framework of our model.). Accordingly, in the main text, we first consider the dynamics of a particle with $\hat{p}$ in the shear plane, which has the significant advantage of being a two-dimensional dynamical system. For a particle with $\hat{p}$ in the shear plane, we define the angle $\xi \in\{-\pi, \pi\}$ to be the angle between the flow direction $\hat{\mathbf{e}}_{y}$ and the particle orientation vector $\hat{p}$. When $-\pi / 2<\xi<\pi / 2$, the particle is oriented downstream; otherwise, the particle is oriented upstream.
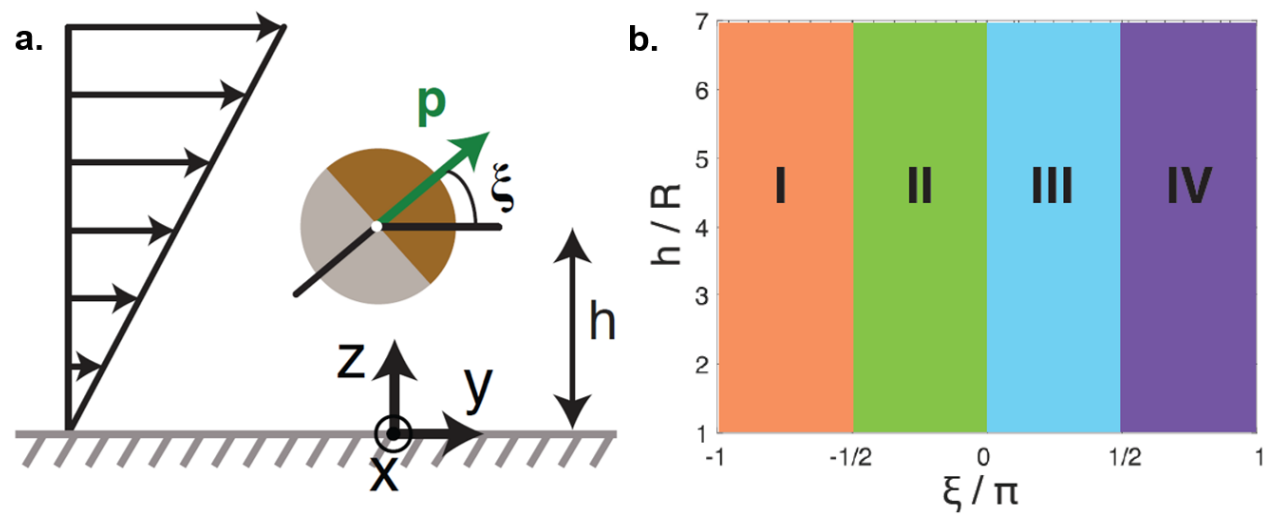

Scheme 2: (a) Definition of the angle $\xi$ for a Janus particle with $\hat{p}$ in the shear plane. The catalytic cap is in black. (b) Definition of four regions of phase space. 


\section{References}

[1] Tolga Kaya and Hur Koser. Direct upstream motility in escherichia coli. Biophysical journal, 102(7):1514-1523, 2012.

[2] Gladys Alexandre, Suzanne Greer-Phillips, and Igor B Zhulin. Ecological role of energy taxis in microorganisms. FEMS microbiology reviews, 28(1):113-126, 2004.

[3] Benjamin M Friedrich and Frank Jülicher. Chemotaxis of sperm cells. Proceedings of the National Academy of Sciences, 104(33):13256-13261, 2007.

[4] Gáspár Jékely. Evolution of phototaxis. Philosophical Transactions of the Royal Society B: Biological Sciences, 364(1531):2795-2808, 2009.

[5] Lukas Niese, Linlin Wang, Sayan Das, and Juliane Simmchen. Apparent phototaxis enabled by brownian motion. Soft Matter, 16:10585-10590, 2020.

[6] Judith P Armitage. Bacterial tactic responses. Advances in microbial physiology, 41:229_ 289, 1999.

[7] FP Bretherton and Nathaniel Mayer Victor Rothschild. Rheotaxis of spermatozoa. Proceedings of the Royal Society of London. Series B. Biological Sciences, 153(953):490-502, 1961.

[8] Zhuoran Zhang, Jun Liu, Jim Meriano, Changhai Ru, Shaorong Xie, Jun Luo, and Yu Sun. Human sperm rheotaxis: a passive physical process. Scientific reports, 6(1):1-8, 2016.

[9] Nuris Figueroa-Morales, Aramis Rivera, Rodrigo Soto, Anke Lindner, Ernesto Altshuler, and Éric Clément. E. coli "super-contaminates" narrow ducts fostered by broad run-time distribution. Science advances, 6(11):eaay0155, 2020. 
[10] Jérémie Palacci, Stefano Sacanna, Anaïs Abramian, Jérémie Barral, Kasey Hanson, Alexander Y Grosberg, David J Pine, and Paul M Chaikin. Artificial rheotaxis. Science advances, 1(4):e1400214, 2015.

[11] Liqiang Ren, Dekai Zhou, Zhangming Mao, Pengtao Xu, Tony Jun Huang, and Thomas E Mallouk. Rheotaxis of bimetallic micromotors driven by chemical-acoustic hybrid power. ACS nano, 11(10):10591-10598, 2017.

[12] Remmi Baker, Joshua E Kauffman, Abhrajit Laskar, Oleg E Shklyaev, Mykhailo Potomkin, Leonardo Dominguez-Rubio, Henry Shum, Yareslie Cruz-Rivera, Igor S Aranson, Anna C Balazs, et al. Fight the flow: the role of shear in artificial rheotaxis for individual and collective motion. Nanoscale, 11(22):10944-10951, 2019.

[13] Quentin Brosseau, Florencio Balboa Usabiaga, Enkeleida Lushi, Yang Wu, Leif Ristroph, Jun Zhang, Michael Ward, and Michael J. Shelley. Relating rheotaxis and hydrodynamic actuation using asymmetric gold-platinum phoretic rods. Phys. Rev. Lett., 123:178004, Oct 2019 .

[14] Arnold JTM Mathijssen, Nuris Figueroa-Morales, Gaspard Junot, Éric Clément, Anke Lindner, and Andreas Zöttll. Oscillatory surface rheotaxis of swimming e. coli bacteria. Nature communications, 10(1):1-12, 2019.

[15] Kai Qi, Hemalatha Annepu, Gerhard Gompper, and Roland G. Winkler. Rheotaxis of spheroidal squirmers in microchannel flow: Interplay of shape, hydrodynamics, active stress, and thermal fluctuations. Phys. Rev. Research, 2:033275, Aug 2020.

[16] Prateek Dwivedi, Atishay Shrivastava, Dipin Pillai, and Rahul Mangal. Rheotaxis of active droplets. Physics of Fluids, 33(8):082108, 2021. 
[17] Ranabir Dey, Carola M Buness, Babak Vajdi Hokmabad, Chenyu Jin, and Corinna C Maass. Oscillatory rheotaxis of active droplets in microchannels. arXiv preprint arXiv:2106.10222, 2021.

[18] Andreas Zöttl and Holger Stark. Nonlinear dynamics of a microswimmer in poiseuille flow. Physical review letters, 108(21):218104, 2012.

[19] WE Uspal, Mikhail N Popescu, S Dietrich, and M Tasinkevych. Rheotaxis of spherical active particles near a planar wall. Soft matter, 11(33):6613-6632, 2015.

[20] J L Anderson. Colloid transport by interfacial forces. Annual Review of Fluid Mechanics, 21(1):61-99, 1989.

[21] Mihail N. Popescu, William E. Uspal, Clemens Bechinger, and Peer Fischer. Chemotaxis of active janus nanoparticles. Nano Letters, 18(9):5345-5349, 2018.

[22] S. Ebbens, D. A. Gregory, G. Dunderdale, J. R. Howse, Y. Ibrahim, T. B. Liverpool, and R. Golestanian. Electrokinetic effects in catalytic platinum-insulator janus swimmers. Europhysics Letters, 106(58003), 2014.

[23] Aidan T. Brown, Wilson C. K. Poon, Christian Holm, and Joost de Graaf. Ionic screening and dissociation are crucial for understanding chemical self-propulsion in polar solvents. Soft Matter, 13:1200-1222, 2017.

[24] Xuemao Zhou, Shuo Wang, Longbin Xian, Zameer Hussain Shah, Yurou Li, Guanhua Lin, and Yongxiang Gao. Ionic effects in ionic diffusiophoresis in chemically driven active colloids. Phys. Rev. Lett., 127:168001, Oct 2021.

[25] Saverio E. Spagnolie and Eric Lauga. Hydrodynamics of self-propulsion near a bound- 
ary: predictions and accuracy of far-field approximations. Journal of Fluid Mechanics, 700:105-147, 2012.

[26] Jonathan R Howse, Richard AL Jones, Anthony J Ryan, Tim Gough, Reza Vafabakhsh, and Ramin Golestanian. Self-motile colloidal particles: from directed propulsion to random walk. Physical review letters, 99(4):048102, 2007.

[27] Andrew I Campbell, Stephen J Ebbens, Pierre Illien, and Ramin Golestanian. Experimental observation of flow fields around active janus spheres. Nature communications, 10(1):1-8, 2019.

[28] Aidan T. Brown, Ioana D. Vladescu, Angela Dawson, Teun Vissers, Jana Schwarz-Linek, Juho S. Lintuvuori, and Wilson C. K. Poon. Swimming in a crystal. Soft Matter, 12:131140, 2016.

[29] Jaideep Katuri, William E Uspal, Juliane Simmchen, Albert Miguel-López, and Samuel Sánchez. Cross-stream migration of active particles. Science advances, 4(1):eaao1755, 2018.

[30] Bishwa Ranjan Si, Preet Patel, and Rahul Mangal. Self-propelled janus colloids in shear flow. Langmuir, 36(40):11888-11898, 2020.

[31] Xianglong Lyu, Xiaoxia Liu, Chao Zhou, Shifang Duan, Pengzhao Xu, Jia Dai, Xiaowen Chen, Yixin Peng, Donghao Cui, Jinyao Tang, Xing Ma, and Wei Wang. Active, yet little mobility: Asymmetric decomposition of $\mathrm{h} 2 \mathrm{o} 2$ is not sufficient in propelling catalytic micromotors. Journal of the American Chemical Society, 143(31):12154-12164, 2021.

[32] Juliane Simmchen, Jaideep Katuri, William E Uspal, Mihail N Popescu, Mykola Tasinkevych, and Samuel Sánchez. Topographical pathways guide chemical microswimmers. Nature communications, 7(1):1-9, 2016. 
[33] Linda Feuerstein, Carl Georg Biermann, Zuyao Xiao, Christian Holm, and Juliane Simmchen. Highly efficient active colloids driven by galvanic exchange reactions. Journal of the American Chemical Society, 2021.

[34] Quentin Brosseau, Florencio Balboa Usabiaga, Enkeleida Lushi, Yang Wu, Leif Ristroph, Michael D. Ward, Michael J. Shelley, and Jun Zhang. Metallic microswimmers driven up the wall by gravity. Soft Matter, 17:6597-6602, 2021.

[35] Felix Rühle, Johannes Blaschke, Jan-Timm Kuhr, and Holger Stark. Gravity-induced dynamics of a squirmer microswimmer in wall proximity. New Journal of Physics, 20:025003, 2018.

[36] Chang Liu, Chao Zhou, Wei Wang, and H. P. Zhang. Bimetallic microswimmers speed up in confining channels. Phys. Rev. Lett., 117:198001, Nov 2016.

[37] Andrew I. Campbell and Stephen J. Ebbens. Gravitaxis in spherical janus swimming devices. Langmuir, 29(46):14066-14073, 2013. PMID: 24134682.

\section{Acknowledgments}

JS, PS and ZX thank the the Volkswagen foundation for financial support in form of a Freigeist grant (No 91619) the Fulbright Cottrell award. PS is also thankful to Graduate Academy, supported by Federal and State Funds. ZX acknowledges the China Scholarship Council. WU and VM gratefully acknowledge the Donors of the American Chemical Society Petroleum Research Fund for support of this research, grant number 60809-DNI9. 


\section{Supplementary materials}

List of supporting videos

Setup used for flow experiments

Zetapotential measurements 